\title{
The Variation in the Lumbar Facet Joint Orientation in an Adult Asian Population and Its Relationship with the Cross-Sectional Area of the Multifidus and Erector Spinae
}

\author{
Kazuhiro Sugawara, Masaki Katayose, Kota Watanabe \\ Graduate School of Health Science, Sapporo Medical University, Sapporo, Japan
}

\begin{abstract}
Study Design: Cross-sectional study of healthy volunteers.
Purpose: We aimed to investigate the variation in the lumbar facet joint orientation in an adult Asian population. The relationship between the facet joint orientation and muscle cross-sectional area (CSA) of multifidus and erector spinae was also clarified.

Overview of Literature: Several studies have reported that lumbar pathologies, such as lumbar spondylolysis and degenerative spondylolisthesis, were related to the horizontally shaped lumbar facet joint orientation at the lower lumbar level. However, data regarding variations in the facet joint orientation in asymptomatic subjects have not been well documented.

Methods: In 31 healthy male adult Asian volunteers, the facet joint orientation and CSA of multifidus and erector spinae were measured using magnetic resonance imaging at the L4-5 and L5-S1 levels. Variation in the facet joint orientation was examined using coefficients of variation (CV). Pearson's product-moment coefficient was used to investigate the relationship between the facet joint orientation and CSA of multifidus and erector spinae.

Results: Lumbar facet joint orientation had a wider range of variation at L5-S1 (CV=0.30) than at $L 4-5$ (CV=0.18). The $L 4-5$ facet joint orientation had a weak but significant correlation with the CSA of erector spinae $(r=0.40 ; p=0.031)$. The CSA of the multifidus had no relationship with the facet joint orientation at the $L 4-5(r=0.19 ; p=0.314)$ and the $L 5-S 1$ level $(r=0.19 ; p=0.312)$.

Conclusions: The lumbar facet joint orientation was found to have a wide variation, particularly at the L5-S1 in the Asian adult population, and the facet joint orientation had a relationship with the CSA of the erector spinae at the L4-5.
\end{abstract}

Keywords: Lumbar vertebrae; Zygapophyseal joint; Paraspinal muscles; Magnetic resonance imaging; Healthy volunteers

\section{Introduction}

Lumbar facet joint determines vertebral motion and transfers upper body weight to the lower body [1]. In the lower lumbar vertebrae, the force acting on the lumbar facet joint becomes greater, and lumbar pathologies, such as spondylolysis and degenerative spondylolisthesis, frequently occur at that level.

Lumbar facet joint orientation has been measured with various methods using bone models [2], magnetic resonance imaging (MRI) $[3,4]$, and computed tomography [5-7]. Facet joint orientation in the sagittal plane has been

\footnotetext{
Received Jan 13, 2016; Revised Apr 24, 2016; Accepted Apr 27, 2016

Corresponding author: Kazuhiro Sugawara

Graduate School of Health Science, Sapporo Medical University, S1 W17, Chuo-ku, Sapporo, 060-8556, Japan

Tel: +81-11-611-2111 (Ext 2967), Fax: +81-11-611-2139, E-mail: k.sugawara@sapmed.ac.jp
} 
reported to be from $150^{\circ}$ to $170^{\circ}$ from the vertical axis with no differences between each lumbar level [2]. In contrast, facet joint orientation in the horizontal plane has been reported as having $10^{\circ}$ to $15^{\circ}$ of standard deviation in Caucasian and African-American bone models [2] and patients with spondylolisthesis in Asia-Pacific legion [8], which suggests a wide variation between individuals. Furthermore, facet joint orientation in the horizontal plane changes toward the sagittal direction with aging $[6,9]$. Several studies have reported that lumbar pathologies, such as lumbar spondylolysis and degenerative spondylolisthesis, were related to the facet joint orientation at the lower lumbar level $[3,8,10]$. However, the data regarding variations in the facet joint orientation in asymptomatic subjects have not been well documented, and additional asymptomatic subject data is important for understanding the normal variation in the facet joint orientation.

The significance in facet joint orientation lies in that it determines vertebral motion and might affect muscle activity around the lumbar vertebrae. Change in the facet joint orientation affects vertebral anterior translation and rotation between adjacent vertebral segments. Erector spinae can rotate the vertebrae and prevent anterior translation by producing posterior force. Lumbar multifidus maintains lumbar lordosis and generates compressive forces with minimal associated torque [11]. As such, the role or function of erector spinae and multifidus might be affected by the facet joint orientation in the horizontal plane. However, the relationship between these muscle functions and orientation of the lumbar facet joints is unclear. We hypothesized that sagittally facing facet joint orientation require support from the erector spinae for controlling vertebral anterior translation. Daily use of the erector spinae affected by lumbar facet joints might appear in the muscle cross-sectional area (CSA) and have a relationship with the facet joint orientation.

We aimed to investigate the variation in the facet joint orientation in the horizontal plane in a healthy adult Asian population and to clarify the relationship between the facet orientation and muscle CSA of the multifidus and erector spinae.

\section{Materials and Methods}

This was a cross-sectional study.

\section{Subjects}

From July 2009 to August 2009, 31 healthy male volunteers among university students participated in this study. Subjects had a mean height of $173.5 \pm 6.2 \mathrm{~cm}$, a mean weight of $64.6 \pm 7.8 \mathrm{~kg}$, and an age range of 20-28 years (mean age, 23.6 years; standard deviation [SD], 1.7 years). This study was approved by the ethics committee of Sapporo Medical University and informed consent was obtained from all subjects.

The inclusion criteria were as follows: (1) body mass index range of $18.5-25 \mathrm{~kg} / \mathrm{m}^{2}$; (2) no participation in competitive sports; (3) no training of the back muscles in the preceding year; and (4) no history and participation in heavy physical work. The exclusion criteria were as follows: (1) a history of lumbar disc herniation, spondylolysis, or spondylolisthesis; (2) a prior surgery of the lumbar spine; and (3) a diagnosis of scoliosis, lower back pain, or use of medication for lower back pain. All of the subjects were examined about abnormal features by a radiologist using MRI.

\section{Protocol for MRI measurements}

The facet joint orientation and the CSA of the back muscles were measured using MRI, as the evaluation of the facet joint orientation using MRI has been validated $[12,13]$.

Subjects were first screened for contraindications to MRI by a radiologist. All MRI examinations were performed using a 1.5 Tesla MR system (Signa HDx, GE HealthCare, Little Chalfont, UK). The subjects were positioned supine, lying with their hips and knees slightly flexed and resting on a foam wedge. The T-1 weighted fast spin echo images (TR/TE $620 / 8.4 \mathrm{~ms}, 3 \mathrm{~mm}$ thick slices, $0.3 \mathrm{~mm}$ interslice spacing, $180 \mathrm{~mm}$ FOV, 256×256 matrix) were then collected. MRI images were taken at the L4-5 and L5-S1 levels. A line parallel to the inferior endplate of L4 was used as a reference line to take the MRI images at L4-5. At the L5-S1 level, a line parallel to the inferior endplate of L5 was used as the reference line. Ten images were taken from the middle of the upper vertebral body to the lower vertebral body. Sagittal images were also taken to confirm the slice level for analysis. The MRI images were saved in the DICOM data format. The orientation of the facet joints and CSA of the back muscles were measured using image analysis software (OsiriX medical 
imaging software, OsiriX, Atlanta, GA, USA).

\section{Measurements of the facet joint orientation}

The measurements of facet joint orientation were according to the procedure followed by Grobler et al. [5]. The facet joint orientation was defined as the angle between the line drawn from the posterior edge of the lumbar body or disc and the line connecting the most medial and lateral edges of the superior facet joint (Fig. 1). The facet joint orientation was measured on MRI parallel to the superior endplate of the lower vertebra at each lumbar level.

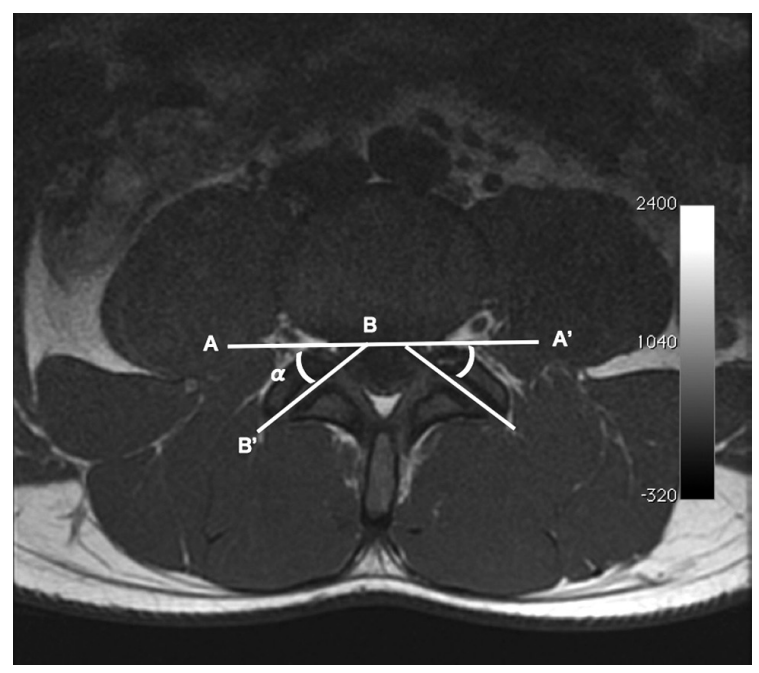

Fig. 1. Measurement of the facet joint orientation. The angle between the line drawn from the posterior edge of the lumbar body or disc $\left(A-A^{\prime}\right)$ and the line connecting the most medial edge of the superior facet joint and the most lateral edge of the superior facet joint $\left(B-B^{\prime}\right)$ was measured on the magnetic resonance image.
Measurements were taken three times in each image, and the average was used for analysis.

\section{Measurements of the CSA of the multifidus and erector spinae}

The lower back muscles were divided into the multifidus and erector spinae; the erector spinae muscles consisted of iliocostalis lumborum and longissimus thoracis. The CSA of the erector spinae was measured where the image disappeared through the transverse process of the lower vertebra at each level and to measure how the erector spinae acted on the upper vertebrae. The CSA of the multifidus was measured where the image disappeared through the spinous process of the upper vertebra at each level, in order to measure how the multifidus acted on the upper vertebrae. The image of the measurements is shown in Fig. 2. An interactive pen display was used to measure the orientation of the facet joint and CSA of the back muscles. Measurements were taken three times, and the average was used for the analysis. Normalization of the CSA of both muscles using body weight and body mass index was highly affected by the body fat. Therefore, the CSAs of the multifidus and erector spinae were divided by the CSA of the L5 vertebral body to normalize the values.

\section{Statistical analysis}

The intra-rater reliability of the measurements of the CSA and the facet joint orientation at the L4-5 and the L5-S1 levels were determined by repeated measurements

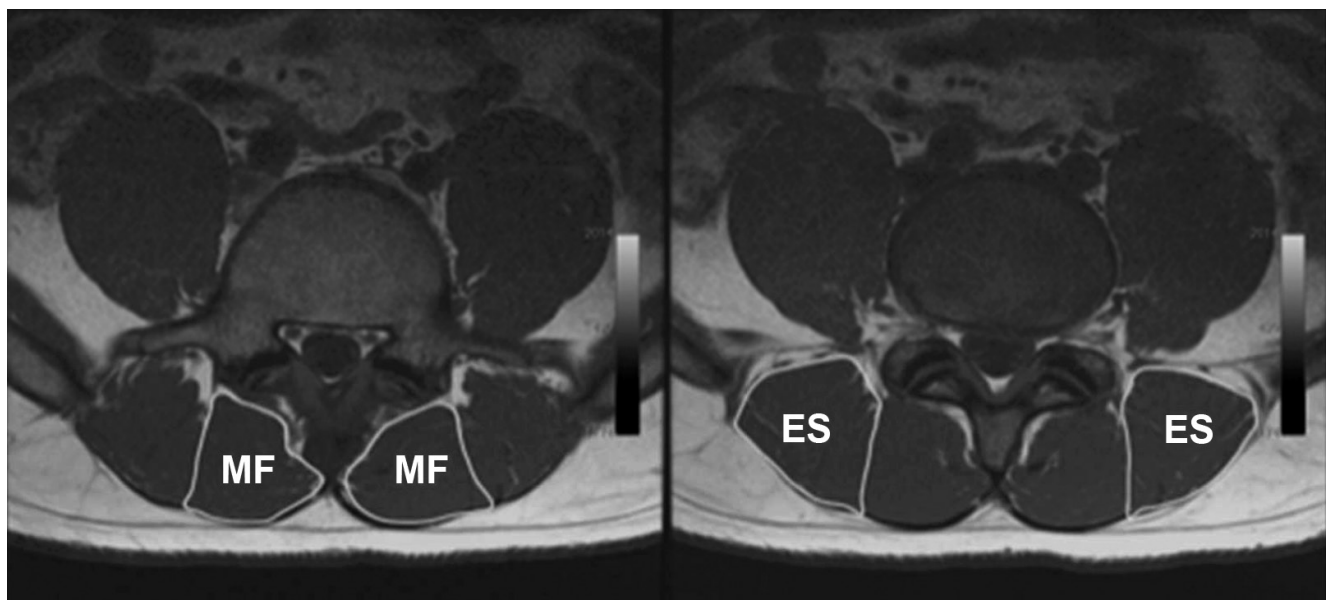

Fig. 2. Measurement of the cross-sectional area of the multifidus and erector spinae on magnetic resonance imaging. MF, multifidus muscle; ES, lumbar erector spinae. 
performed one week apart by the same examiner in the 31 subjects. The order of the images was randomized at the second measurements, and the results of the first measurements were blinded. Intra-rater reliability of the measurement of the CSA and the facet joint orientation were examined using intraclass correlation coefficients that were examined in a one-way classification with absolute agreement with $95 \%$ confidence intervals for intrarater reliability.

The facet joint orientation and the CSAs of the multifidus and erector spinae at each level are reported as mean \pm SD. The variations in the facet joint orientation are expressed by the coefficients of variation. Masuyama's rejection limit test was used to examine outliers in CSA of erector spinae and multifidus. Pearson's product-moment coefficient was used to analyze the relationship between the facet joint orientation and CSA of the erector spinae and multifidus. Right-side data of the facet joint orientation and CSA of erector spinae and multifidus were used for correlation analysis. Statistical significance was set at $p<0.05$.

\section{Results}

\section{Reliability of measurement methods}

The intraclass correlation coefficient was high for both the measurements of the facet joint orientation (intraclass correlation coefficient $=0.958 ; 95 \%$ confidence interval $=0.938-0.973)$ and the measurements of the CSA of the multifidus (intraclass correlation coefficient $=0.979$; 95\% confidence interval $=0.967-0.986)$ and erector spinae (intraclass correlation coefficient $=0.972 ; 95 \%$ confidence interval $=0.952-0.982$ ).

\section{Variation in the facet joint orientation}

One result of the L5-S1 level was excluded because the facet joints were not visible enough to measure the facet joint orientation. The total numbers of facet joints for analysis were 62 at the L4-5 and 60 at the L5-S1. The angle of the facet joint orientation was not statistically different between the right side and the left side at each level ( $p>0.05)$. The coefficient of variation for the L5-S1 facet joint orientation was larger than that for $\mathrm{L} 4-5$, as shown in Table 1 and Fig. 3 (L5-S1: coefficient of variation=0.30; L4-5: coefficient of variation $=0.18$ ).

\section{Relationship between the facet joint orientation and CSA of the multifidus and erector spinae}

The CSA and normalized values of the multifidus and

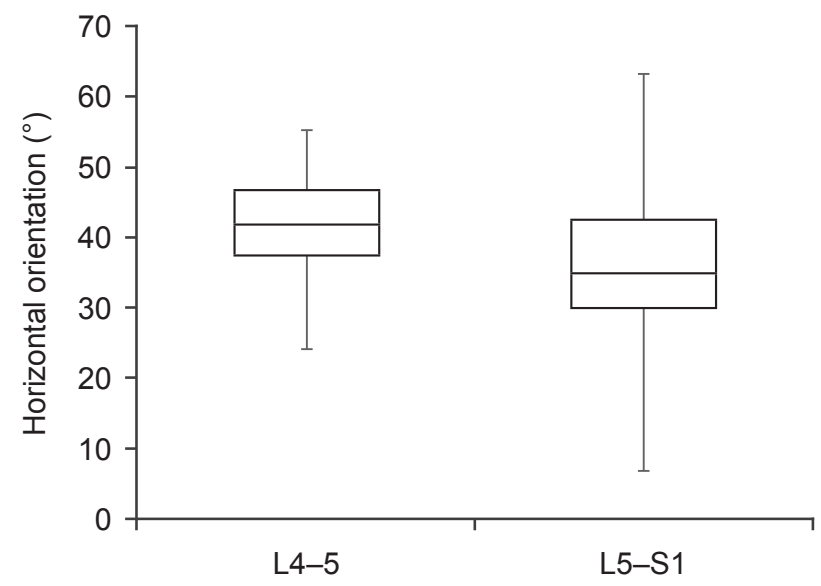

Fig. 3. Box plots for the facet joint orientation at the $L 4-5$ and the $L 5-$ S1 levels.

Table 1. Facet joint orientation at the $L 4-5$ and the $L 5-S 1$ level

\begin{tabular}{|c|c|c|c|c|c|}
\hline Vertebral level and side & Average $\left({ }^{\circ}\right)$ & $\operatorname{SD}\left({ }^{\circ}\right)$ & $\operatorname{Max}\left({ }^{\circ}\right)$ & $\operatorname{Min}\left({ }^{\circ}\right)$ & CV \\
\hline \multicolumn{6}{|l|}{ L4-5 } \\
\hline Right & 41.1 & 7.5 & 53.7 & 24.1 & 0.18 \\
\hline Left & 41.4 & 7.6 & 55.2 & 26.4 & 0.18 \\
\hline Total & 41.3 & 7.5 & 55.2 & 24.1 & 0.18 \\
\hline \multicolumn{6}{|l|}{ L5-S1 } \\
\hline Right & 34.8 & 11.9 & 63.2 & 6.8 & 0.34 \\
\hline Left & 35.4 & 9.6 & 53.2 & 10.9 & 0.27 \\
\hline Total & 35.1 & 10.7 & 63.2 & 6.8 & 0.30 \\
\hline
\end{tabular}

SD, standard deviation; Max, maximum value; Min, minimum value; CV, coefficients of variation. 
Table 2. Cross-sectional area of the multifidus and erector spinae

\begin{tabular}{|c|c|c|c|c|}
\hline \multirow{2}{*}{ Muscles } & \multicolumn{2}{|c|}{$\mathrm{CSA}\left(\mathrm{cm}^{2}\right)$} & \multicolumn{2}{|c|}{ Normalized value } \\
\hline & Right side & Left side & Right side & Left side \\
\hline \multicolumn{5}{|l|}{ Multifidus } \\
\hline L4 & $8.9 \pm 1.6$ & $8.6 \pm 1.6$ & $0.51 \pm 0.11$ & $0.49 \pm 0.10$ \\
\hline $\mathrm{L} 5$ & $8.3 \pm 1.7$ & $8.2 \pm 1.6$ & $0.47 \pm 0.10$ & $0.47 \pm 0.10$ \\
\hline \multicolumn{5}{|c|}{ Erector spinae } \\
\hline Ł4 & $11.1 \pm 1.8$ & $12.0 \pm 2.1$ & $0.63 \pm 0.10$ & $0.69 \pm 0.13$ \\
\hline $\mathrm{L} 5$ & $8.2 \pm 2.4$ & $8.9 \pm 2.5$ & $0.46 \pm 0.14$ & $0.51 \pm 0.16$ \\
\hline
\end{tabular}

CSA, cross-sectional area.
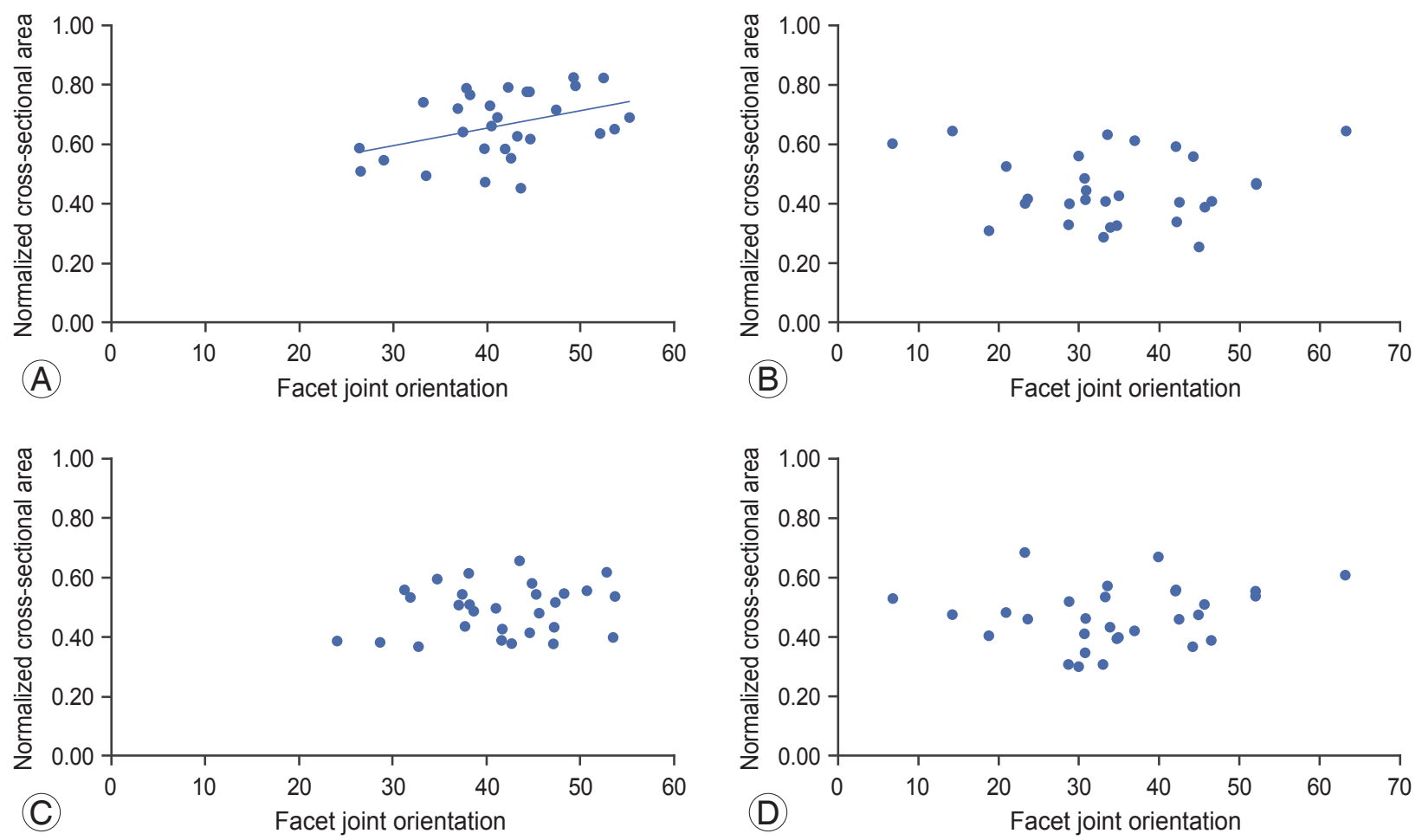

Fig. 4. Relationship between the facet joint orientation and the CSA of each muscle on the right side. (A) Erector spinae at $L 4-5$. (B) Erector spinae at L5-S1. (C) Multifidus at L4-5. (D) Multifidus at L5-S1. CSA, cross-sectional area.

erector spinae at L4 and L5 are shown in Table 2. Rejection limit for normalized CSA of multifidus was $0.28-0.74$ for L4 and 0.26-0.68 for L5. Rejection limit for normalized CSA of erector spinae was $0.42-0.84$ for L4 and 0.17-0.75 for L5. Two data of both multifidus and erector spinae at L4-5 level and one data of erector spinae at L5-S1 were excluded for correlation analysis because of them exceeding the rejection limit of CSA. The facet joint orientation at L4-5 had a weak but significant correlation with the CSA of the erector spinae at L4 $(r=0.40, p=0.031$, $1-\beta=0.62$ ) (Fig. 4A). The CSA of the erector spinae had no relationship with the facet joint orientation at the L5$\mathrm{S} 1(\mathrm{r}=-0.024, \mathrm{p}=0.900$ ) (Fig. 4B). The CSA of the multifidus had no relationship with the facet joint orientation at the L4-5 ( $r=0.19 ; p=0.314$ ) (Fig. 4C) and the L5-S1 level $(r=0.19 ; p=0.31)$ (Fig. 4D).

\section{Discussion}

Our study had two major findings. First, in a healthy adult 
Asian population, the facet joint orientation of the L5-S1 had a wider variation than that of the L4-5. Second, the facet joint orientation at the L4-5 had a weak relationship with the CSA of the erector spinae in healthy population.

Grobler et al. [5] studied the facet joint orientation at the L4-5 in a healthy population who were approximately 40 years in age and reported an orientation of the left and right lumbar facet joint of $44.91^{\circ} \pm 10.83^{\circ}$ and $41.09^{\circ} \pm 10.35^{\circ}$, respectively. In contrast, Wang and Yang [6] reported an average facet joint orientation at the L4-5 of $49.41^{\circ} \pm 5.29^{\circ}$ in healthy males under 30 years of age. Our results of the L4-5 facet joint orientation do not correspond to Wang et al's report and indicate a variation between individuals in the same adult Asian population. Moreover, our finding of the facet joint orientation at the L5-S1 of $35.1^{\circ} \pm 10.7^{\circ}$ is relatively different from the findings of Grobler et al. [5], i.e. $47.62^{\circ} \pm 11.15^{\circ}$ for the left and $42.91^{\circ} \pm 11.81^{\circ}$ for the right lumbar facet joints. Several studies have reported that the facet joint orientation becomes more sagittal with age $[6,9]$. Therefore, the difference in the results for the facet joint orientation at the L5-S1 in the healthy population between our study and Grobler's might be explained by the difference in age of the two study populations. Williams et al. [8] reported an average facet joint orientation at the L5-S1 of $43.3^{\circ} \pm 13.0^{\circ}$ for the left and $43.1^{\circ} \pm 12.5^{\circ}$ for the right lumbar facet joints in patients with degenerative spondylolisthesis in a multicenter study in the Asia-Pacific region. The difference in the results for the facet joint orientation at the L5-S1 between our study and Williams et al's might be explained by the presence of degenerative spondylolisthesis and difference in the age of the two study populations. Our results show a great amount of variation in the facet joint orientation at the L5-S1 and could be a part of asymptomatic subject data in the Asian adult population.

Coefficient of variation is dependent on the mean size and standard deviation. From our results, the mean size of the L5-S1 was smaller than that of the L4-5 and standard deviation of the L5-S1 was greater than L4-5 level. Although both the mean size and standard deviation affected the greater value of CV at the L5-S1 level, the boxplot showed that the L5-S1 had a greater amount of variation than that for the L4-5 level. The greater amount of variation in the facet joint orientation of the L5-S1 may relate to the characteristics of lumbar movements at this level between individuals. It is important to understand such variability when evaluating the mobility of the lumbar vertebrae. For example, we can speculate on the limitation in lumbar flexion-extension mobility from images of frontally faced facet joint orientation in MRI.

The hypothesis of this study was that the CSA of the erector spinae has correlated with the facet joint orientation, as a more sagittal facet joint orientation requires support from the erector spinae in preventing vertebral anterior translation. Our results show that the more frontal facet joint orientation at the L4-5 has less CSA of the erector spinae than the more sagittal facet joint orientation. This result suggests that the more sagittal facet joint orientation in a healthy population related to increasing erector spinae muscle CSA, so vertebral anterior translation might be controlled. Grobler et al. [5] reported that the lumbar degenerative spondylolisthesis patients had more sagittal facet joint orientation at L4-5 than the control subjects. Further studies are needed to clarify the relationship between the facet joint orientation and the CSA of erector spinae in lumbar pathology such as degenerative spondylolisthesis, and our results could be compared in such a study.

The facet joint orientation at the L5-S1 level had no relationship with the CSA of the erector spinae. This was because the L5 vertebra has the iliolumbar ligament, which restricts vertebral motion in any direction. Also, the multifidus did not have any relationship with the horizontal facet orientation. This reflects the function of the multifidus in stabilizing the lumbar spine independent of the direction of movement $[14,15]$.

There were several limitations to this study. First, all of the subjects in this study were only male and under 30 years of age. Therefore, the results couldn't justify the applicability to additional age groups and the female gender in the Asian population and those would be a part of this population data. Second, lumbar facet joints have a threedimensional construction; however, they were only evaluated from one MRI slice in this study. Future research is required to evaluate the facet joint orientation threedimensionally and to clarify the effect of horizontal orientation on the muscle activity of the multifidus and erector spinae.

\section{Conclusions}

In conclusion, the lumbar facet joint orientation was found to have a wide variation particularly at the L5-S1 in the Asian adult population, and the facet joint orientation 
had a relationship with the erector spinae at the L4-5.

\section{Conflict of Interest}

No potential conflict of interest relevant to this article was reported.

\section{References}

1. Bogduk N. Clinical anatomy of the lumbar spine and sacrum. 3rd ed. New York: Churchill Livingstone; 1997.

2. Masharawi Y, Rothschild B, Dar G, et al. Facet orientation in the thoracolumbar spine: three-dimensional anatomic and biomechanical analysis. Spine (Phila Pa 1976) 2004;29:1755-63.

3. Don AS, Robertson PA. Facet joint orientation in spondylolysis and isthmic spondylolisthesis. J Spinal Disord Tech 2008;21:112-5.

4. Masharawi Y, Kjaer P, Bendix T, et al. The reproducibility of quantitative measurements in lumbar magnetic resonance imaging of children from the general population. Spine (Phila Pa 1976) 2008;33:2094-100.

5. Grobler LJ, Robertson PA, Novotny JE, Pope MH. Etiology of spondylolisthesis. Assessment of the role played by lumbar facet joint morphology. Spine (Phila Pa 1976) 1993;18:80-91.

6. Wang J, Yang X. Age-related changes in the orientation of lumbar facet joints. Spine (Phila Pa 1976) 2009; 34:E596-8.

7. Chung SB, Lee S, Kim H, Lee SH, Kim ES, Eoh W. Significance of interfacet distance, facet joint orientation, and lumbar lordosis in spondylolysis. Clin Anat
2012;25:391-7.

8. Williams R, Cheung JP, Goss B, et al. An International Multicenter Study Assessing the Role of Ethnicity on Variation of Lumbar Facet Joint Orientation and the Occurrence of Degenerative Spondylolisthesis in Asia Pacific: A Study from the AOSpine Asia Pacific Research Collaboration Consortium. Global Spine J 2016;6:35-45.

9. Masharawi YM, Kjaer P, Bendix T, et al. Lumbar facet and interfacet shape variation during growth in children from the general population: a three-year follow-up MRI study. Spine (Phila Pa 1976) 2009;34: 408-12.

10. Masharawi Y, Dar G, Peleg S, et al. Lumbar facet anatomy changes in spondylolysis: a comparative skeletal study. Eur Spine J 2007;16:993-9.

11. Bogduk N, Macintosh JE, Pearcy MJ. A universal model of the lumbar back muscles in the upright position. Spine (Phila Pa 1976) 1992;17:897-913.

12. Boden SD, Riew KD, Yamaguchi K, Branch TP, Schellinger D, Wiesel SW. Orientation of the lumbar facet joints: association with degenerative disc disease. J Bone Joint Surg Am 1996;78:403-11.

13. Dai LY. Orientation and tropism of lumbar facet joints in degenerative spondylolisthesis. Int Orthop 2001;25:40-2.

14. MacDonald DA, Moseley GL, Hodges PW. The lumbar multifidus: does the evidence support clinical beliefs? Man Ther 2006;11:254-63.

15. Richardson C. Therapeutic exercise for spinal segmental stabilization in low back pain: scientific basis and clinical approach. Edinburgh: Churchill Livingstone; 1999. 